\title{
CRITERIOS PARA ANALIZAR EL USO DE INTERNET EN INSTITUCIONES UNIVERSITARIAS
}

Resumen: En el siguiente artículo presentamos los resultados obtenidos en un trabajo de investigación bibliográfico dirigido a organizar los criterios de la clasificación utilizados para analizar las instituciones universitarias dependiendo del uso que hacen de Internet. Tras comprobar que los criterios vigentes se pueden agrupar en torno a dos ejes (ámbitos de uso y grado de presencialidad-virtualidad), proponemos la creación de un nuevo eje centrado en aspectos didácticos. Esto nos permitirá determinar no sólo los ámbitos y el grado de incorporación de Internet, sino también si se dan las condiciones necesarias para afrontar los cambios pedagógicos que la UNESCO propone en su "Declaración de la Educación Superior en el Siglo XXI" y que resultan necesarios para afrontar con éxito el Espacio Europeo de la Educación Superior. Todos los datos aquí presentados se enmarcan en una investigación de carácter documental y empírica realizada en la Universidad del País Vasco.

Palabras clave: educación superior, Enseñanza universitaria, nuevas tecnologías de la información y la comunicación, Internet, didáctica.

\section{LES CRITERES POUR ANALYSER L'USAGE D'INTERNET DES INSTITU- TIONS UNIVERSITAIRES}

Sommarie: Dans l'article suivant nous présentons les résultats obtenus dans un travail bibliographique d'investigation dirigé à organiser les critères de classification utilisés pour analyser l'usage d'Internet des institutions universitaires. Après avoir vérifié que les critères en vigueur peuvent se grouper autour à deux axes (l'usage et le niveau de présence-virtualité), nous proposons la création d'un nouvel axe focalisé sur des éléments didactiques. Cela nous permettra de déterminer non seulement les enceintes et le degré d'incorporation d'Internet, mais aussi si existent les conditions nécessaires pour affronter les échanges pédagogiques que l'UNESCO propose dans sa "Déclaration de l'Education Supérieure au XXIe siècle" et qu'ils sont nécessaires pour affronter avec succès l'Espace Européen de l'Education Supérieure. Toutes les données ici présentés sont encadrées dans une investigation de caractère documentaire et empirique réalisée dans l'Université du Pays Basque.

Mots clés : éducation supérieure, enseignement universitaire, nouvelles technologies de l'information et de la communication, Internet, didactique.

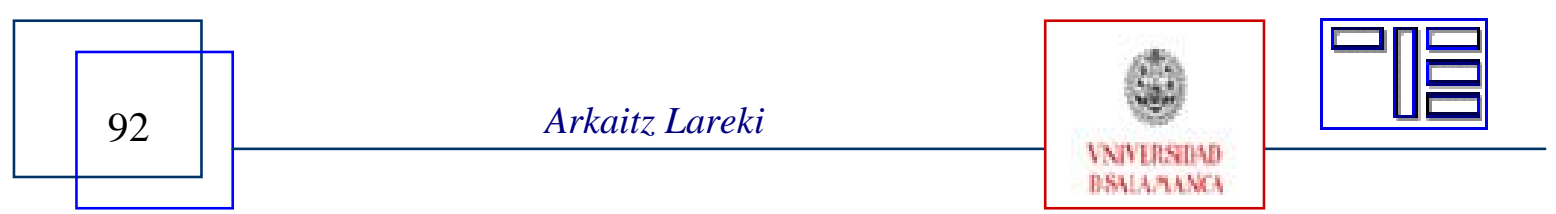




\section{CRITERIONS TO ANALYZE INTERNET USE AT UNIVERSITY INSTITU- TIONS}

\section{Abstract:}

This Article presents a literature review focus on the organization of the classification criteria used to analyze the dependence on the use of Internet at university institutions. After checking that, the actual criterions can be divided in two activity points (use fields and presence-virtual degree). In this study also a new activity point focus on didactic aspect was proposed. This way, not only the fields and the incorporation degree could be determined, also could be possible to afford the pedagogical changes proposed by the UNESCO on XXI Century High Education Statement, which are necessary to face successfully High Education in the European Area. All the data presented in this document was obtained by a broad documentation on the field and also by empiric way at the University of the Basque Country.

Key words: high education, new technologies on information and communication, internet, didactic.

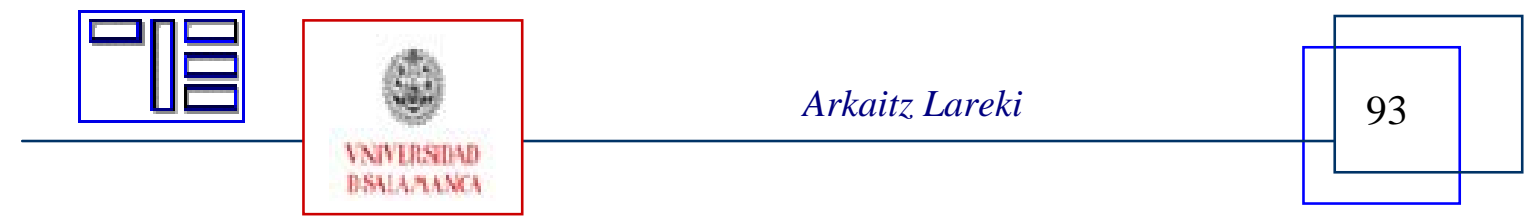


Revista Electrónica Teoría de la Educación.

Educación y Cultura en la Sociedad de la Información.

http://www.usal.es/teoriaeducacion

Vol. 9. No 1. Febrero 2008

\title{
CRITERIOS PARA ANALIZAR EL USO DE INTERNET EN INSTITUCIONES UNIVERSITARIAS
}

\author{
Arkaitz Lareki \\ Arkaitz.lareki@ehu.es \\ Universidad del País Vasco
}

Las nuevas tecnologías de la información y de la comunicación, y en concreto el uso de Internet entendido como máximo representante de estas tecnologías, han reportado ciertas ventajas e inconvenientes (Area, 2000; Cabero, 2002; Salinas 2002; Gisbert, 2004) que han repercutido en la estructura y organización de estos centros de educación superior, y especialmente en las condiciones de los procesos de enseñanza-aprendizaje que en ellas se llevan a cabo.

Asimismo, durante los últimos años en la medida en que cada nueva tecnología se ha empezado a utilizar en la educación se han tratado de establecer las bases para su evaluación, clasificación o análisis ${ }^{1}$. La enseñanza universitaria mediada por Internet no escapa a esta realidad. Para afrontar con éxito procesos de análisis sobre el uso de Internet en la educación universitaria será necesario, al igual que en el resto de los casos, establecer unos criterios sólidos y suficientemente amplios que nos permitan llevar a cabo un diagnóstico no sesgado, ya que sólo así podremos establecer las bases para realizar una comparación entre los usos que se realizan en diferentes instituciones de enseñanza superior.

\section{Clasificación de las universidades atendiendo al ámbito y grado de incorporación de Internet.}

Hoy en día la extensión de Internet en centros de educación superior universitarios es una realidad, pero dependiendo de las decisiones que cada institución hace en torno a los ámbitos que van a copar su uso, podemos obtener diferentes modelos de universidades agrupados en seis categorías distintas (Duart, 1998). Tomando esta clasificación por buena, podemos perfilar los rasgos generales de cada uno de estos tipos:

Un primer grupo lo conforman las universidades tradicionales que introducen elementos de virtualidad en su dinámica organizativa. Es decir, instituciones que sin incorporar la red como recurso educativo sí hacen un uso administrativo de la misma. Ésta bien puede servir para agilizar la comunicación entre distintos departamentos, para publicitarse a través de la web... etc.

Un segundo grupo lo forman las universidades tradicionales que introducen elementos de virtualidad en su dinámica educativa. Los profesores disponen de los recursos materiales necesarios (ordenadores) y una infraestructura básica (Intranet - Internet) que

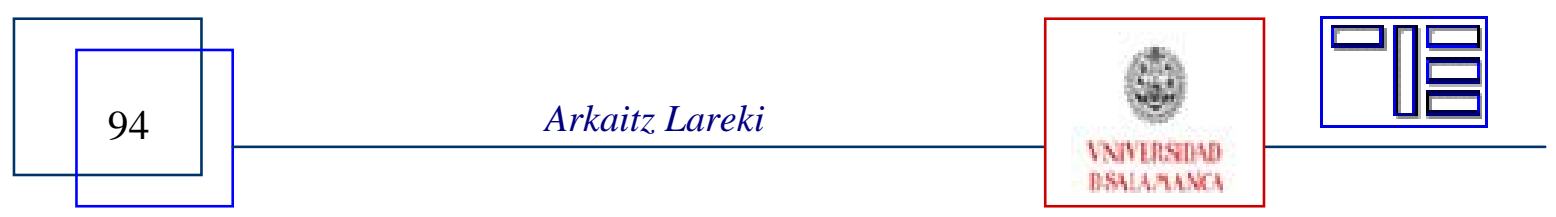


permite la realización de actividades en las que la red se muestra como un recurso didáctico añadido. El desarrollo de estas actividades puede implicar que el alumno y profesor compartan la misma aula o que cada uno desde su ordenador participe sin la necesidad de coincidir ni en el espacio ni el tiempo.

El tercer grupo estaría formado por las universidades tradiciones que, además de ofrecer la infraestructura necesaria para red y dar la posibilidad a los profesores y alumnos de utilizarla como recurso, generan una extensión virtual que sirve como estructura básica para posibilitar el encuentro en ese mismo espacio virtual entre alumnos, profesores, personal de administración y servicios, investigadores, becarios... Es decir, se da no sólo una infraestructura física suficiente (red más ordenadores), sino también una infraestructura virtual básica (entorno) mediante la oferta de servicios: alojamiento web, chat, foro, correo electrónico... etc.

Un cuarto modelo de utilización de Internet por las universidades, es la aparición en una universidad tradicional presencial de una extensión que además de soporte físico y virtual, ofrece la posibilidad de realizar estudios a través del campus virtual. Es decir, ofrece, aparte de la enseñanza presencial, módulos de enseñanza a distancia por ordenador. Por lo tanto habrá instituciones que solo oferten módulos concretos de asignaturas, otras que den la posibilidad de cursar una titulación completa, y otras que den la posibilidad de cursas créditos optativos o de libre elección. Las universidades que adoptan este modelo no abandonan su estructura presencial.

En la quinta categoría encontramos las universidades que son consideradas completamente virtuales y que no disponen de una infraestructura física (universidad como organización virtual.). Aunque por trámites administrativos tengan su sede en una punto geográfico determinado, éstas carecen de aulas, bibliotecas... a las que se tengan que desplazarse los alumnos. En estos casos, tanto la enseñanza como la tramitación de las gestiones puramente administrativas también se realizarían mediante la red. Entendemos que las universidades que han desarrollado este modelo son las nuevas instituciones de educación superior de reciente creación y aquellas que ofreciendo enseñanza a distancia se han adaptado para ofrecer sus servicios a través de la red.

Un sexto grupo estaría formado por aquellas instituciones que ofrecen enseñanza virtual a distancia y que se unen para formar un campus interuniversitario o metacampus. Esta categoría no tendría como unidad básica el modelo que una universidad adopta en la incorporación de Internet sino el grupo de universidades que unidas forman una red de enseñanza virtual superior.

Los altos niveles de incorporación de Internet en las universidades que ofrecen enseñanza presencial y aquellas que ofrecen enseñanza virtual disponen, cuando menos, de una estructura virtual lo suficientemente estable como para dar servicio a sus alumnos; y muchas son las que, manteniendo su presencialidad, ofrecen paquetes de enseñanza que se pueden cursar vía web. Esto les sitúa entre las categorías tres y cuatro.

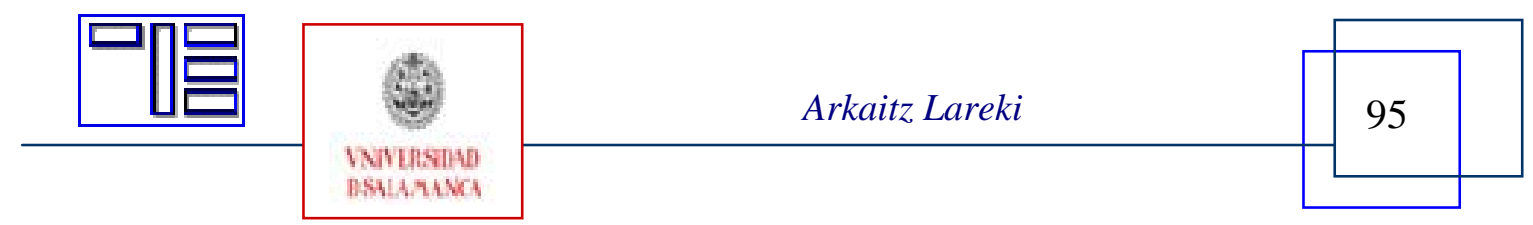


Al hilo de las clasificaciones que se pueden hacer según el grado de penetración de Internet en los distintos ámbitos de acción de las universidades (tareas docentes y administrativas), existen otros criterios que también pueden resultar útiles a la hora de determinar el nivel de integración de Internet, y cuya responsabilidad recae tanto en los órganos colegiados que establecen las estrategias marco de adquisición de recursos tecnológicos en cada institución como en el propio profesorado. En este sentido Area (2000) propone otra clasificación que debe ser entendida como un continuo que va de lo sencillo a lo complejo: desde la utilización de la red como un recurso en la enseñanza presencial a su integración en la educación virtual.

El primer nivel de incorporación de Internet es la edición de documentos convencionales en HTML. De forma que los mismos documentos que son utilizados en la práctica docente presencial (apuntes, ejercicios, programa...) se puedan colgar en la red para que los alumnos lo tengan a su disposición.

Le sigue la elaboración de materiales didácticos electrónicos o tutoriales para la web para que junto con los materiales de utilizados en el aula se puedan generar nuevos materiales basados y pensados para ser utilizados en formato digital, con todo lo que esto conlleva (hipertexto, navegar por la web... etc.).

El tercer nivel es el diseño y desarrollo de cursos on-line semipresenciales que compaginen clases presenciales con sesiones vía Internet en donde los alumnos utilicen recursos específicos generados a tal efecto.

En el último nivel se encuentra la educación virtual como sistema educativo que ofrece a los alumnos la capacidad de desarrollar aprendizajes por medio de materiales y recursos dispuestos en la red por el profesorado, de manera que la comunicación entre ambos agentes esté mediada siempre por el ordenador.

Observamos que esta clasificación tiene una coincidencia implícita con el siguiente cuadro de Duart y Sangrá (2000) que clasifica los modelos de enseñanza universitaria que integran Internet atendiendo a la coincidencia en el espacio y en el tiempo. Así el primer nivel equivaldría al primer cuadrante y el último al cuarto.

\begin{tabular}{|l|l|l|}
\hline & Coincidencia en el tiempo & No coincidencia en el tiempo \\
\hline $\begin{array}{l}\text { Coincidencia en el } \\
\text { espacio }\end{array}$ & Formación presencial & $\begin{array}{l}\text { Autoaprendizaje en centro de } \\
\text { recursos } \\
\text { Teleaprendizaje }\end{array}$ \\
\hline No coincidencia en el & Enseñanza por radiodifusión & Formación no presencial \\
\hline
\end{tabular}

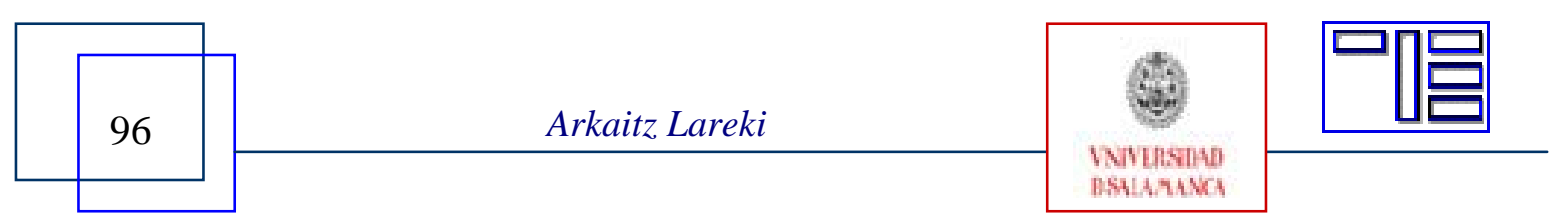




\begin{tabular}{|l|l|l|}
\hline espacio & Televisión educativa & $\begin{array}{l}\text { Enseñanza por correspondencia } \\
\text { Entornos virtuales de aprendizaje } \\
\text { (universidad virtual) }\end{array}$ \\
\hline
\end{tabular}

1. Cuadro: Cuadrante de espacio y de tiempo en la formación (Duart y Sangrá, 2000).

Para que una universidad de carácter presencial pueda alcanzar el nivel que se plantea como objetivo en cualquiera de las escalas que hemos visto, es imprescindible que afronte la secuencia descrita por Sangrá y González Sanmamed (2004). Aunque la consecución de las fases no tendría por qué ser necesariamente consecutiva ni excluyente se aprecia un orden lógico que, según estos autores, sitúa en el primer lugar la fase de equipamiento mediante la cual se dota a los agentes de la universidad de los recursos necesarios que permitirán el uso de Internet en la educación superior. Una vez adquirida la tecnología se requiere una la capacitación tecnológica de los agentes (docentes y discentes) que un contexto educativo van a hacer uso de los recursos puestos a su alcance, y que a la postre mediarán en la labor de enseñanza de los profesores y la tarea de aprendizaje de los alumnos.

Pocas son, según Sangrá y González Sanmamed (2004), las instituciones que han alcanzado la tercera fase, y que se caracteriza por estar centrada primordialmente en el docente. En ella, se capacita pedagógicamente a los profesores con el fin de que lleven a cabo un correcto uso didáctico de las tecnologías de la información y la comunicación que permita la obtención de los objetivos educativos esperados.

Por último, será necesaria una revisión del planteamiento y del programa seguido para llevar a cabo la incorporación de las nuevas tecnologías. A través de esta evaluación se debe revisar tanto los programas de dotación de equipos como los planes de trabajo y las practicas educativas que han resultado ser más adecuadas para incorporar las nuevas tecnologías.

La consecución de todos los pasos descritos debería permitir a cada universidad alcanzar el modelo educativo que quiera lograr, bien sea dirigiendo toda su docencia a través de clases on-line a distancia, bien reforzando la clase presencial mediante el uso de recursos tecnológicos como sistema de apoyo, creando para ello aulas específicas de acceso a Internet o haciendo llegar la red a las aulas tradicionales.

Atendiendo a los parámetros que aquí hemos visto, el modelo que ha prevalecido para incorporar Internet a la enseñanza superior se ha dirigido a generar programas de educación a virtual distancia, de manera que un gran número de universidades tradicionalmente presenciales han incorporado paquetes formativos de enseñanza virtual a través de Internet $^{2}$. Muchas de aquellas universidades que han hecho inversiones para abarcar este nuevo mercado, se han encontrado con problemas similares a los que surgieron cuando se implantó la enseñanza por correspondencia. De manera que las fuertes inver-

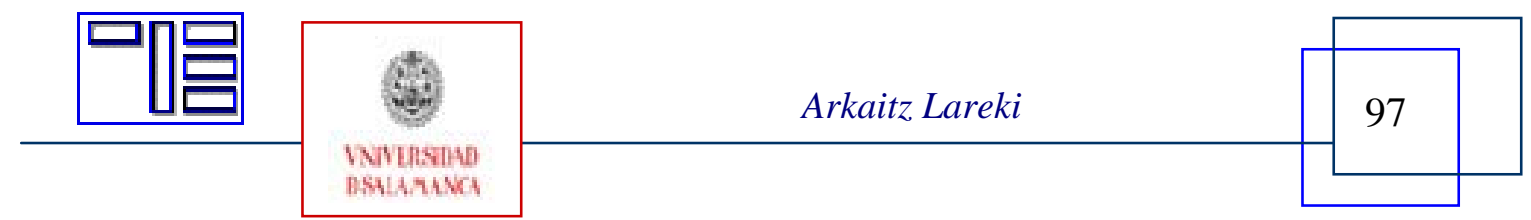


siones realizadas en recursos técnicos y organizativos, en personal docente y de mantenimiento, no han dado los frutos esperados, y estas instituciones han acumulado grandes pérdidas económicas ${ }^{3}$.

\section{La necesidad de un nuevo eje de análisis: el aspecto pedagógico.}

En 1998 la UNESCO a través de su Declaración Mundial de la Educación Superior en el siglo XXI advertía que las universidades presenciales tradicionales debían buscar el máximo aprovechamiento de las nuevas tecnologías de la información y comunicación para mejorar las condiciones de la enseñanza superior y no para entrar en una lucha por copar el mercado de la teleformación ${ }^{4}$. Esta referencia hay que entenderla como una clara invitación a mejorar las condiciones didáctico-pedagógicas de la enseñanza que se ofrece desde las universidades. En relación al trabajo que aquí presentamos este aspecto es el que genera el tercer eje sobre el que se agrupan los criterios de análisis de las universidades en relación al uso de Internet. Este eje se añadiría a los dos que hemos visto en el apartado anterior

Enseñanza Universitaria

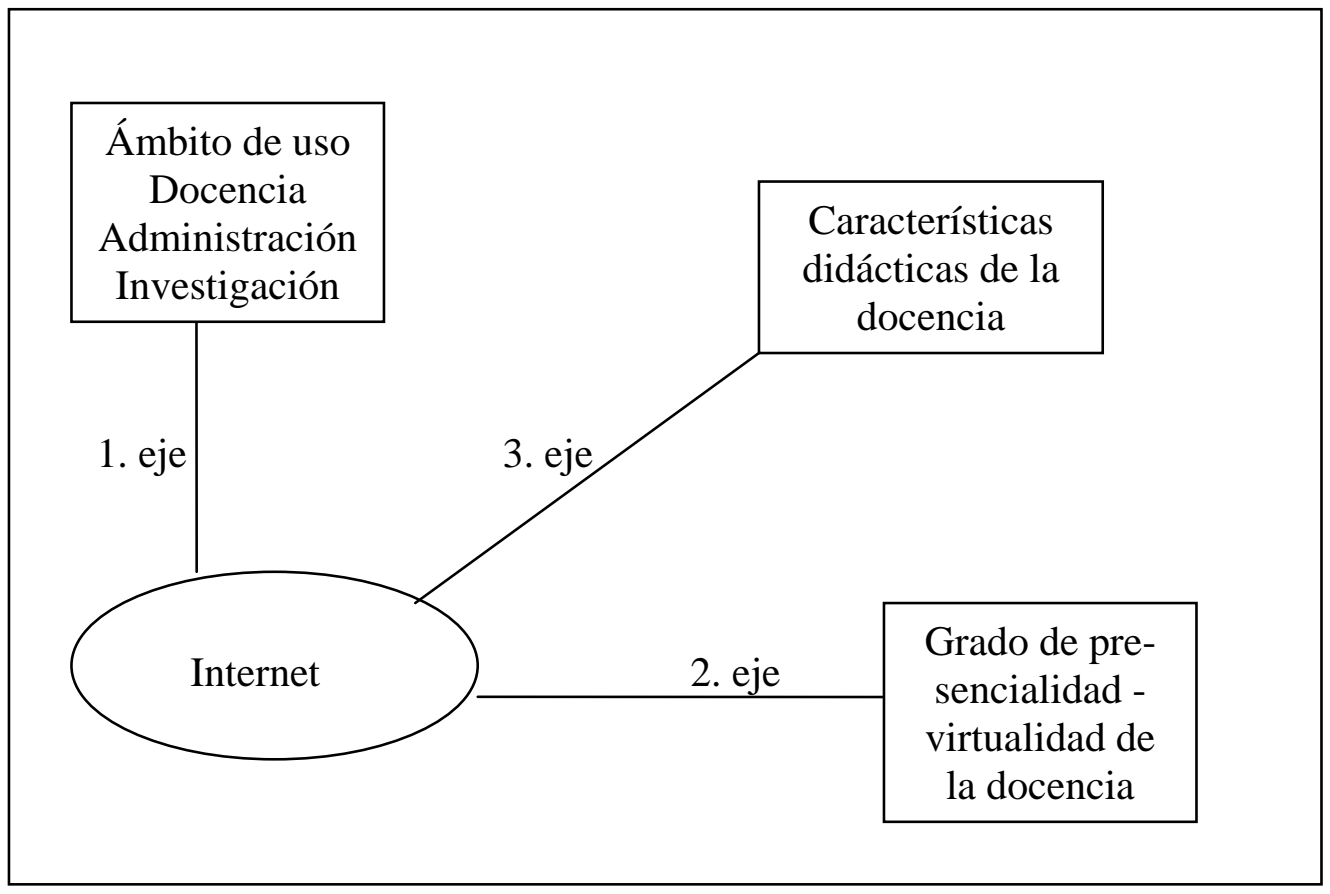

2. Cuadro: Ejes de análisis del uso de Internet en enseñanza universitaria.

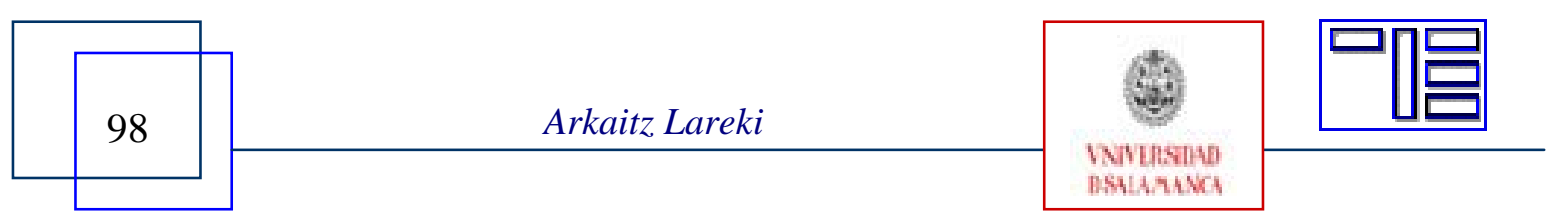


El análisis del uso de Internet en contextos de enseñanza, por lo tanto, se ha de ajustar a un diseño tridimensional. En primer lugar se estudiarán los ámbitos que se ven afectados al incorporar Internet (administración, docencia y gestión), seguidos del grado de presencialidad y virtualidad que adquiere la docencia (segundo eje). En tercer lugar se han de estudiar las características didácticas y pedagógicas que tiene la enseñanza en la que se producen los usos de Internet.

Profundizando en este tercer eje, entendemos que el objeto de estudio que posibilitará el análisis será el conjunto de tareas o actividades que vertebran la acción educativa, puesto que en ellas se producirán los usos educativos de Internet. Conocer estas tareas se convierte, por lo tanto, en un requisito necesario. El estudio de los diferentes tareas que desarrollan los docentes que imparten en educación superior ha sido realizado por diversos autores (Zabalza, 2003; Benito A. y De Cruz, 2005; De Miguel Díaz, 2006) generalmente desde la perspectiva metodológica. Destacamos la síntesis presentada por Goñi (2005) quien establece un listado de 23 tipos de tareas diferentes. A saber:

1. Clase expositiva

2. Tareas de aplicación o ejercicios

3. Respuestas a cuestionarios

4. Seminario

5. Prácticas de laboratorio

6. Resolución de problemas

7. Resolución de conflictos/dilemas

8. Audición/visionado de audio/vídeo

9. Lectura y recensión de libros

10. Trabajos de investigación

11. Debates

12. Simulaciones / juegos de rol / dramatizaciones

13. Presentación y defensa de un tema, trabajo investigación.

14. Realización de informes memorias diarios

15. Observación sistemática, recogida de datos, copia de modelos

16. Análisis de situaciones documentos, productos estudio de caso

17. Estudio sistematizado

18. Desarrollo escrito de un tema

19. Realización de entrevistas, encuestas

20. Visitas guiadas

21. Proyectos

22. exámenes.

23. Otras.

El análisis en torno al tercer eje no ha de centrarse sólo en las tareas en las que se contextualiza el uso de Internet - ya que esto nos llevaría a una visión incompleta de la realidad - sino que habrá que tener una visión global del tipo de tareas que se proponen durante el tiempo que dura todo el proceso formativo. Conocidas el conjunto de tareas y

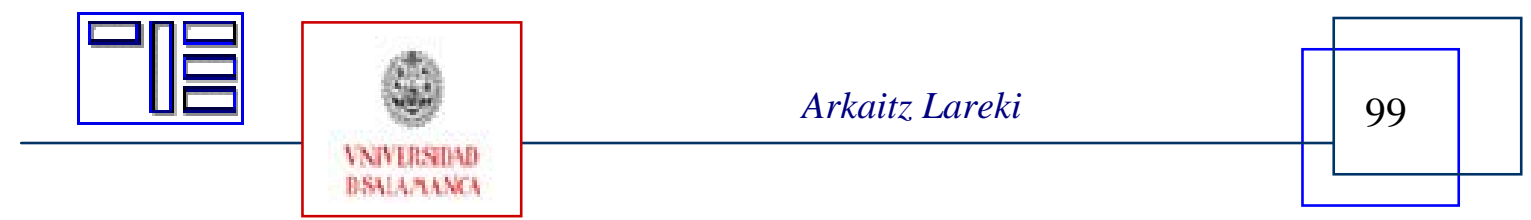


detectadas aquellas en las que se focaliza la utilización de Internet, habrá que pasar al análisis pormenorizado de los distintos aspectos pedagógicos. Para ello, cada uno de los criterios va acompañado de un grado de variabilidad que pretende guiar y facilitar el análisis:

\begin{tabular}{|l|l|}
\hline Criterios & Grado de variabilidad \\
\hline Rol del profesor & $\begin{array}{l}\text { El profesor se establece como poseedor exclusivo del cono- } \\
\text { cimiento y se erige en el transmisor de la información que ha } \\
\text { de asimilar el alumno. } \\
\text { El profesor propone actividades adecuadas que permiten la } \\
\text { adquisición de conocimientos, aumentando así su papel de } \\
\text { diseñador y gestor de actividades y guía de los aprendizajes. }\end{array}$ \\
\hline Rol del alumno & $\begin{array}{l}\text { El alumno se convierte en receptor de la información transmi- } \\
\text { tida por el docente, y a aquel concierne la asimilación de esta } \\
\text { información. } \\
\text { La participación del alumno en el desarrollo de las distintas } \\
\text { tareas resulta fundamental para adquirir los aprendizajes. Se } \\
\text { fomenta una actitud activa de búsqueda y reconstrucción de la } \\
\text { información que le permita adquirir nuevos conocimientos. }\end{array}$ \\
\hline $\begin{array}{l}\text { Concepción del curri- } \\
\text { culum }\end{array}$ & $\begin{array}{l}\text { Existe un temario oficial cerrado que ha de ser transmitido a } \\
\text { todo los alumnos por igual. } \\
\text { Además de los contenidos conceptuales (temario) se da espe- } \\
\text { cial importancia a la movilización adecuada de esos conteni- } \\
\text { dos para afrontar con éxito ciertas tareas (competencias). } \\
\text { El enfoque curricular bajo el que se estructura la asignatura es } \\
\text { flexible, lo que permite el trabajo de cierto número de com- } \\
\text { petencias diferenciadas dependiendo de los intereses de los } \\
\text { alumnos. } \\
\text { Los contenidos de las materias se ajustan a las características } \\
\text { del entorno para que los alumnos puedan adquirir competen- } \\
\text { cias útiles en ese contexto social. }\end{array}$ \\
\hline $\begin{array}{l}\text { La adquisición de los conocimientos se produce, principal- } \\
\text { mente, por la repetición de los contenidos. } \\
\text { Se parte de los conocimientos que tienen los alumnos para } \\
\text { proponer actividades adecuadas que permitan adquirir nuevos } \\
\text { conocimientos. } \\
\text { Se fomenta que el aprendizaje se adquiera, también, a través }\end{array}$ \\
\hline
\end{tabular}

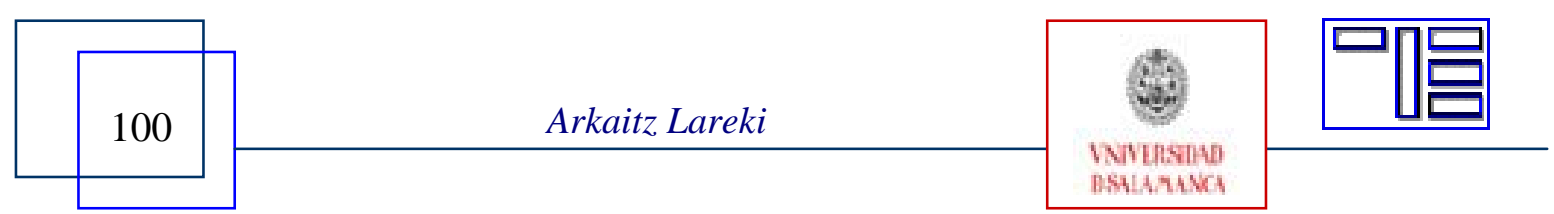




\begin{tabular}{|l|l|}
\hline & $\begin{array}{l}\text { de la colaboración entre sujetos. } \\
\text { Se reflexiona sobre el proceso y estrategias seguidas para la } \\
\text { adquisición de conocimientos nuevos. }\end{array}$ \\
\hline $\begin{array}{l}\text { Sistemas de evalua- } \\
\text { ción. }\end{array}$ & $\begin{array}{l}\text { La evaluación está dirigida básicamente a la comprobación de } \\
\text { los aprendizajes del alumno, por lo que se establecen sistemas } \\
\text { de evaluación final. } \\
\text { La evaluación adquiere un carácter formativo, para lo cual se } \\
\text { establecen sistemas de evaluación continua que permita un } \\
\text { feedback entre el profesor y el alumno. }\end{array}$ \\
\hline
\end{tabular}

3. Cuadro: Criterios pedagógicos para el análisis del uso de Internet en la universidad.

Los criterios y el grado de variabilidad que hemos recogido en este cuadro son aplicables tanto a modelos de enseñanza virtual, presencial o mixto en el que se utilice Internet. Esto implica que modelos muy distintos pueden ser muy iguales un punto de vista didáctico-pedagógico; y viceversa, modelos aparentemente muy iguales pueden ser didáctica y pedagógicamente radicalmente diferentes. Entendemos, de esta manera, que la incorporación de Internet en la enseñanza universitaria no implica necesariamente cambios pedagógicos significativos, si bien es cierto que entre Internet y educación surge una relación compleja semejante a la que describe Castells (1996) entre tecnología y sociedad.

Para finalizar conviene recordar que, en el caso que nos ocupa, el estudio de las tareas no es un fin en sí mismo, sino el medio que nos va a permitir estudiar las características didácticas y pedagógicas en las que se contextualiza la utilización de Internet. A través de ellas profundizaremos en aspectos como: el rol que desempeña el profesorado en los procesos formativos en los que interviene, el rol que adquiere el alumno, la concepción del curriculum que guía la práctica educativa, los principios y teorías psicopedagógicos que intervienen en la propuesta formativa y el sistema de evaluación empleado. Un análisis pormenorizado de estos criterios atendiendo a su grado variabilidad, unido a un análisis en torno al primer y segundo eje, nos permitirá recoger una imagen bastante completa de la realidad de uso de Internet en diferentes universidades.

\section{3-. REFERENCIAS}

AREA, M. (2000): ¿Qué aporta Internet al cambio pedagógico en la educación? en R. PÉREZ (Coord): Redes multimedia y diseños virtuales. Actas del III Congreso Internacional de Comunicación, Tecnología y Educación. Universidad de Oviedo, septiembre 2000,. 128-135.

BARTOLOMÉ, A (2004): Blended learning. Conceptos básico. Revista Pixel-bit Revista de Medios y Educación, 23, 7-20.

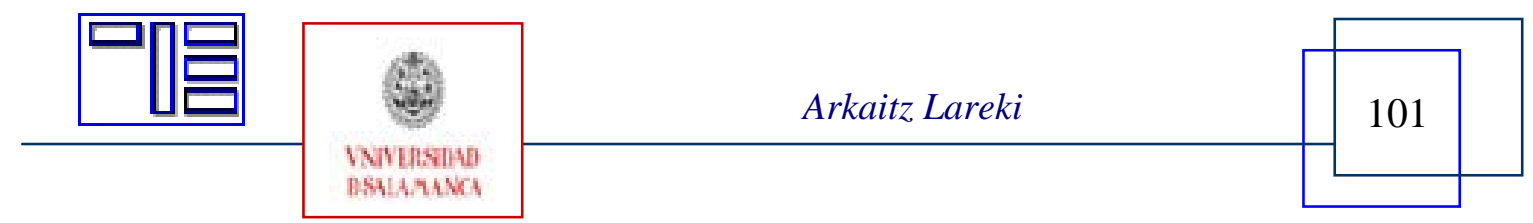


Revista Electrónica Teoría de la Educación.

Educación y Cultura en la Sociedad de la Información.

http://www.usal.es/teoriaeducacion

Vol. 9. No 1. Febrero 2008

BENITO, A. y CRUZ, A. (2005): Nuevas claves para la docencia universitaria en el espacio europeo de educación superior . Madrid, Narcea.

CABERO, J. (2002): Las TICs en la Universidad. Sevilla, MAD.

CASTELLS, M. (1996) La Sociedad Red. Madrid, Alianza.

DE MIGUEL DÍAZ, M. (Dir.) (2006): Metodologías de enseñanza y aprendizaje para el desarrollo de competencias. Orientaciones para el profesorado universitario ante el Espacio Europeo de la Educación Superior. Madrid, Alianza.

DUART, J. M. (1998): Aulas y campus virtuales I y II. Avances en Tecnología Educativa. Donostia, Universidad de Verano del País Vasco.

DUART, J. M y SANGRÁ, A. (2000): Aprender en la virtualidad. Barcelona, Gedisa.

GISBERT, M. (2004): Las TIC como motor de innovación en la universidad. En SANGRÁ Y GONZÁLEZ SANMAMED (coords.): La transformación de las universidades a través de las TIC: discursos y prácticas. Barcelona, UOC.

GOÑI, J. M. (2005): El espacio europeo de educación superior, un reto para la universidad. Barcelona, Octaedro.

MARQUÉS, P. (2000): Nuevos instrumentos para la evaluación de materiales multimedia. Comunicación y pedagogía, 166, 103-117.

PAVÓN, F (2003): El nuevo escenario europeo de educación superior y el papel a desempeñar por las nuevas tecnologías de la información y la comunicación. XXI, Revista de Educación, 5 103-108.

RÍOS, P. (2001) concepción del software educativo desde la perspectiva pedagógica. Quaderns Digitals, 24.

SALINAS, J. (2002): ¿Qué aportan las tecnologías de la información y la comunicación a las universidades convencionales? Algunas consideraciones y reflexiones. Revista Educación y Pedagogía, 33, 91-105.

SANGRÁ, A. y GONZÁLEZ SANMAMED, M (2004): La transformación de las universidades a través de las TICs. Barcelona, UOC.

UNESCO (1998): Declaración mundial sobre la educación superior en el siglo XXI: visión y acción. http://www.unesco.org/education/educprog/wche/declaration_spa.htm UNESCO (2006): Revisión de la competencia como organizadora de los programas de formación: hacia un desempeño competente. Ginebra, IBE-UNESCO.

ZABALZA, J. A. (2003): Competencias docentes del profesorado universitario. Calidad y desarrollo professional. Madrid, Narcea.

\section{Notas}

[ $\left.{ }^{1}\right]$ Ríos (2001) por ejemplo establece los criterios para la construcción de software educativo. Marqués (2000) los fundamentos para la evaluación de portales educativos... etc.

[2] Noble D. F. (1998): La inminente batalla sobre la teleformación. Factorías de diplomas digitales. http://www.zmag.org/Spanish/0011digi.htm [consulta 15/11/2007]

[3] "Hay que hacer notar que cuando el proyecto de aprendizaje digital estaba ligado a la evolución de sistemas de enseñanza a distancia (ED), no se produce este fracaso por cuanto los costo no superan a los de la ED clásica y los porcentajes de alumnos que superan con éxito no son menores que los que había

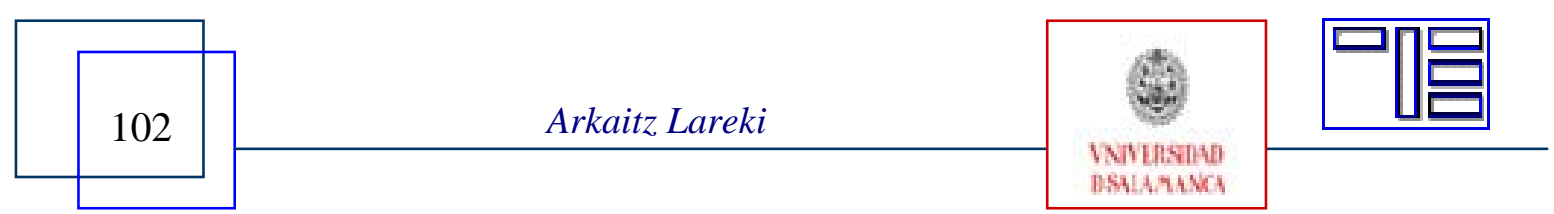


antes. Incluso es posible encontrar algunas estadísticas con datos positivos. Pero cuando se trata de universidades tradicionales que han querido reconvertir sus programas en cursos basados en aprendizaje digital, tanto cursos de pregrado como de postgrado, o de centro de formación presencial que han querido hacer esta transformación, entonces es cuando se produce la debacle. Y es que, volviendo a la discusión sobre modelos anterior, el e-learning es un modelo de organización del aprendizaje adecuado a los sistemas de ED." (Bartolomé 2004:5).

$\left[{ }^{4}\right]$ "Teniendo en cuentas las nuevas posibilidades abiertas por el uso de las tecnologías de la información y la comunicación, es importante observar que ante todo son los establecimientos de educación superior los que utilizan esas tecnologías para modernizar su trabajo en lugar de que éstas transformen a establecimientos reales en entidades virtuales.” (UNESCO 1998).

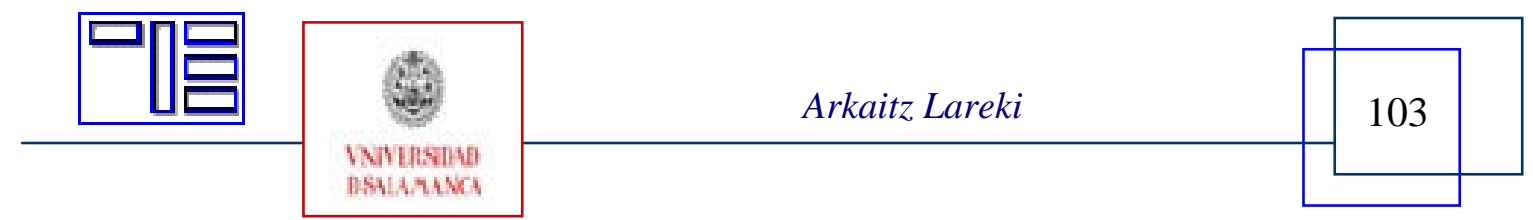

